\title{
Feasibility of Energy Harvesting Using Stochastic Resonance Caused by Axial Periodic Force
}

\author{
Kimihiko Nakano*,1 - Matthew P. Cartmell ${ }^{2}$ - Honggang Hu${ }^{1}$ - Rencheng Zheng1 \\ ${ }_{1}^{1}$ University of Tokyo, Institute of Industrial Science, Japan \\ 2 University of Sheffield, Department of Mechanical Engineering, United Kingdom
}

\begin{abstract}
Stochastic resonance is a physical phenomenon where large vibration occurs when a weak sinusoidal force is applied to a bi-stable system. It is expected that a larger vibrational response can be produced than for a typical resonance. Then the authors utilize this system as an energy harvester, which converts energy from vibration. The energy balance between the converted energy and the energy consumed to produce the necessary weak sinusoidal force is analyzed through numerical simulations. It is shown the proposed harvester can convert more energy than a system using a typical linear system resonance.
\end{abstract}

Keywords: periodic excitation, bi-stable system, energy harvesting, stochastic resonance

\section{INTRODUCTION}

In recent years, the field of energy harvesting has developed strongly and has increasingly become an important application area. Energy harvesting is the process of capturing trace amounts of energy from the environment and transforming them into electrical energy, such as solar power, thermal energy, wind energy, salinity gradients, vibration energy, etc. While solar, chemical and thermal methods are sometimes viable, many researchers have recognized the abundance of vibration as a potential source of energy [1].

Vibration energy can be converted to electric energy using several types of electro-mechanical transducers based on electromagnetism [2], electrostatics [3] and [4] and piezoelectricity [5]. Electro-mechanical transducers are typically realized as linear mechanical resonators. The effectiveness of such converters is maximum when the transducer is operated at resonance, however it can be considerably suboptimal with frequency-varying conditions and wideband vibrations [6]. If the environmental vibration frequency deviates a little from the designed frequency, the generated power decreases rapidly. Some researchers have extended output power through increasing the resonant frequency bandwidth of the harvester [7] and [8]. The harvester with a tunable resonance frequency is also considered as an effective method [9] and [10]. Given the bandwidth limitation of linear transducers, nonlinearity is also considered as a possibility for improving the effectiveness of energy harvesting [11] and [12], mainly because nonlinear systems are capable of responding over a broad frequency range.
The phenomenon of the stochastic resonance requires three basic ingredients: (a) an energetic activation barrier such as the double potential well of a bi-stable system, (b) a weak coherent input such as a periodic signal, and (c) a source of noise that is inherent to the system. Given these features, the response of the system undergoes resonance-like behaviors as a function of the noise level, hence the name stochastic resonance [13]. The original work on stochastic resonance is due to Benzi et al. [14], for explaining the periodic recurrences of the earth's ice ages. Recently, stochastic resonance has been considered in several fields, such as signal processing [15], circuit experiments [16], and image visualization [17], etc. More especially, McInnes et al. illustrated the possibility of the application of stochastic resonance as an enhancing method for vibration energy harvesting, and demonstrated its benefit in theory [18].

For a nonlinear mechanical system, ambient vibration can be considered as source of noise which can excite a stochastically resonant system into a bistable nonlinear response. The presence of bistability makes the system capable of rapidly switching between stable states under an external nonlinear force. So, if a weak parametric excitation is applied to the system the double well potential periodically raises and lowers the potential barrier, and noiseinduced hopping between the potential wells can become synchronised with the parametric excitation, leading to stochastic resonance. On this basis, the stochastic resonance was produced in a cantilever beam where a periodic force is applied in the lateral direction to the beam [19] in the experiments. It was shown the response vibration was enhanced and 
the amount of the energy to be harvested increased through experiments [20].

In this study, the authors propose to apply the periodic force to the axial direction of the beam. Then dynamics of the beam is governed by a periodic excitation system. In the previous system [19] and [20], the periodic force need to be supplied from the system, however, in the proposed system, the stochastic resonance is expected to be produced in the periodic excitation system exposed to a wide bandwidth of weak ambient broadband vibration. The feasibility to cause the stochastic resonance with the periodic force in the axial direction of beam to enhance the vibration energy to be harvested is examined through numerical analysis.

\section{STOCHASTIC RESONANCE}

\subsection{Modeling of the Energy Harvester}

Based on the theory of stochastic resonance a bistable nonlinear vibration energy harvester is conjectured as shown in Fig. 1. The system is mainly composed of a cantilever beam with an end magnet, and another fixed magnet. It is excited by ambient vibration $N(t)$, and there is an interaction between the elastic force of the beam and the magnetic force, generating a nonlinear response. By adjusting the distance $d$ between the two magnets, the system can show bistability. An actuator is used to provide the source of the periodic parametric excitation.

When a piezoelectric material is placed under a mechanical stress, an open circuit voltage appears across the material. Suitable piezoelectric materials for vibration energy harvesting are characterized by the large magnitude of the product of the piezoelectric voltage constant and the piezoelectric strain constant. The material can be in the form of polycrystalline ceramics, textured ceramics, thin films, and polymers. Therefore, in this paper it is considered that two piezoelectric films are fitted onto the cantilever beam to provide the nonlinear vibration energy harvester with a simple but effective mechanical to electrical energy conversion facility.

Due to the configuration of the two magnets, as discussed in [19], a repulsive magnetic force $F_{\mathrm{M}}$ will act between the two magnets. When $F_{\mathrm{M}}$ makes the angle $\theta$ to $x$ axis and the vertical displacement of the end magnet is $y_{\mathrm{M}}$, the vertical component, $F_{\mathrm{V}}$, can be written in terms of $F_{\mathrm{M}}$ and expanded into a Taylor series, computed around $y_{\mathrm{M}}=0$ and truncated as follows,

$$
\begin{aligned}
F_{\mathrm{V}} & =F_{\mathrm{M}} \sin \theta=\frac{F_{\mathrm{M}}}{d} \frac{y_{\mathrm{M}}}{\left[1+\left(y_{\mathrm{M}}^{2} / d^{2}\right)\right]^{1 / 2}} \approx \\
& \approx \frac{F_{\mathrm{M}}}{d} y_{\mathrm{M}}-\frac{F_{\mathrm{M}}}{2 d^{3}} y_{\mathrm{M}}^{3} .
\end{aligned}
$$

a)

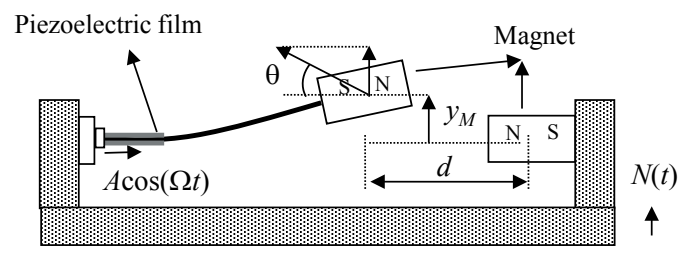

b)

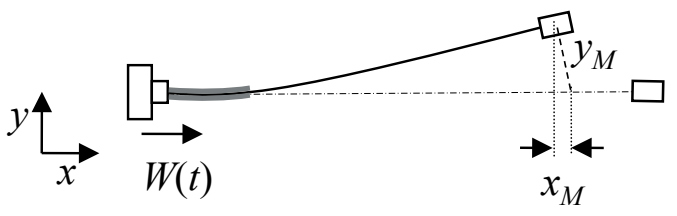

Fig. 1. An energy harvester using stochastic resonance; a) system layout, and b) simple schematic

When the periodic excitation $W(t)$ is input to the system by the actuator in the horizontal direction, and assuming the beam is mass less, the total kinetic energy will be defined by:

$$
E_{k}=\frac{1}{2} m\left[\dot{y}_{\mathrm{M}}^{2}+\left(\dot{x}_{\mathrm{M}}-\dot{W}\right)^{2}\right]
$$

where $x_{\mathrm{M}}$ is the lateral displacement of the end magnet. According to the thin beam theory, the potential energy of the beam whose Young's modulus and moment of inertia are $E$ and $I$, respectively, is given by:

$$
U=\frac{1}{2} E I \int_{0}^{l}\left(w^{\prime \prime}\right)^{2} d x,
$$

where $w$ is the transverse displacement of the beam and $w^{\prime \prime}=d^{2} w / d x^{2}$. Assuming $y_{\mathrm{M}}(t)=w(l, t)$, where $l$ is the length of the beam, the following relationship is derived:

$$
w(x, t)=v(x) w(l, t)=\left(\frac{3 x^{2}}{2 l^{2}}-\frac{x^{3}}{2 l^{3}}\right) y_{\mathrm{M}}(t),
$$

where $v(x)$ is a static linear shape function of the cantilever beam. Therefore the following can be shown from Eqs. (3) and (4):

$$
U=\frac{1}{2} E I\left[\int_{0}^{l}\left(v^{\prime \prime}\right)^{2} d x\right] y_{\mathrm{M}}^{2},
$$


where $v^{\prime \prime}=d^{2} f / d x^{2}$. Assuming the transverse deflection is tiny, the lateral displacement of the end magnet, $x_{\mathrm{M}}$, is given by:

$$
\begin{aligned}
x_{\mathrm{M}} & =\int_{0}^{l}\left[\sqrt{1+\left(\frac{d w}{d x}\right)^{2}}-1\right] d x= \\
& =\int_{0}^{l} \frac{1}{2}\left(\frac{d w}{d x}\right)^{2} d x=\frac{1}{2}\left[\int_{0}^{l}\left(v^{\prime}\right)^{2} d x\right] y_{\mathrm{M}}^{2} .
\end{aligned}
$$

Differentiating Eq. (6) with respect to time leads to:

$$
\dot{x}_{\mathrm{M}}=B y_{\mathrm{M}} \dot{y}_{\mathrm{M}},
$$

where $B=\left[\int_{0}^{l}\left(v^{\prime}\right)^{2} d x\right]$. Substituting Eq. (7) into (2), the kinetic energy can be expressed as:

$$
E_{k}=\frac{1}{2} m \dot{y}_{\mathrm{M}}^{2}+\frac{1}{2} m\left(B^{2} y_{\mathrm{M}}^{2} \dot{y}_{\mathrm{M}}^{2}+\dot{W}^{2}-2 B y_{\mathrm{M}} \dot{y}_{\mathrm{M}} \dot{W}\right) .
$$

According to the generalized Lagrange's equation:

$$
\frac{d}{d t}\left(\frac{\partial E_{k}}{\partial \dot{y}_{\mathrm{M}}}\right)-\frac{\partial E_{k}}{\partial y_{\mathrm{M}}}+\frac{\partial U}{\partial y_{\mathrm{M}}}=F_{\mathrm{V}}
$$

the following equation is derived:

$$
\begin{aligned}
& m \ddot{y}_{\mathrm{M}}\left(1+B^{2} y_{\mathrm{M}}^{2}\right)-m B^{2} y_{\mathrm{M}} \dot{y}_{\mathrm{M}}^{2}+ \\
& +E I\left[\int_{0}^{l}\left(v^{\prime \prime}\right)^{2} d x\right] y_{\mathrm{M}}-m B y_{\mathrm{M}} \ddot{W}=F_{\mathrm{V}} .
\end{aligned}
$$

This is the governing equation for the model proposed. By neglecting the two nonlinear terms, i.e. the second and third left hand side terms, Eq. (10) can be reduced to:

$$
\begin{aligned}
& m \ddot{y}_{\mathrm{M}}+\left(E I\left[\int_{0}^{l}\left(v^{\prime \prime}\right)^{2} d x\right]-\frac{F_{\mathrm{M}}}{d}\right) y_{\mathrm{M}}- \\
& -m B y_{\mathrm{M}} A \Omega^{2} \cos \Omega t+\frac{F_{\mathrm{M}}}{2 d^{3}} y_{\mathrm{M}}^{3}=0,
\end{aligned}
$$

where $W(t)=A \cos (\Omega t)$ is the periodic excitation. By adding in a viscous damping term and inputting an ambient vibration $N(t)$, one obtains;

$\ddot{y}_{\mathrm{M}}+c \dot{y}_{\mathrm{M}}-\left(\omega^{2}+B A \Omega^{2} \cos \Omega t\right) y_{\mathrm{M}}+b y_{\mathrm{M}}^{3}=N(t)$,

where $\quad \omega^{2}=\frac{1}{m}\left\{\frac{F_{\mathrm{M}}}{d}-E I\left[\int_{0}^{l}\left(v^{\prime \prime}\right)^{2} d x\right]\right\} \quad$ and $b=\frac{F_{\mathrm{M}}}{2 m d^{3}}$. Here, $\omega$ is corresponding to the undamped natural frequency of the system. Assuming $W(t)$ is constant periodic force, $B A \Omega^{2}$ can be considered to be constant. When we define $\lambda$ as $B A \Omega^{2} \omega^{-2}$, Eq. (12) can be transformed to:

$$
\ddot{y}_{\mathrm{M}}+c \dot{y}_{\mathrm{M}}-\omega^{2}(1+\lambda \cos \Omega t) y_{\mathrm{M}}+b y_{\mathrm{M}}^{3}=N(t),
$$

which is standard equation describing the system exploring stochastic resonance.

\subsection{Analysis of the Conditions for Stochastic Resonance}

According to the three basic ingredients for stochastic resonance, a weak coherent input such as a periodic signal is required. Although the periodic excitation is too weak to make the system jump periodically from one potential well to the other, the hopping between the potential wells occur when the system disturbed by the noise is synchronized with the periodic excitation, which is the stochastic resonance.

For the model proposed, the weak periodic excitation is provided by the parametric excitation. Consequently, the potential energy can be expressed as:

$$
U\left(y_{\mathrm{M}}, t\right)=-\frac{1}{2} \omega^{2}(1+\lambda \cos \Omega t) y_{\mathrm{M}}^{2}+\frac{1}{4} b y_{\mathrm{M}}^{4} .
$$

Fig. 2 shows the changing potential well as a function of displacement. It can be seen that the height of the potential well is modulated periodically. According to the theory of stochastic resonance, running through the frequency fulfils certain conditions, and this modulation enables the excitation $N(t)$ to drive the system into stochastic resonance.

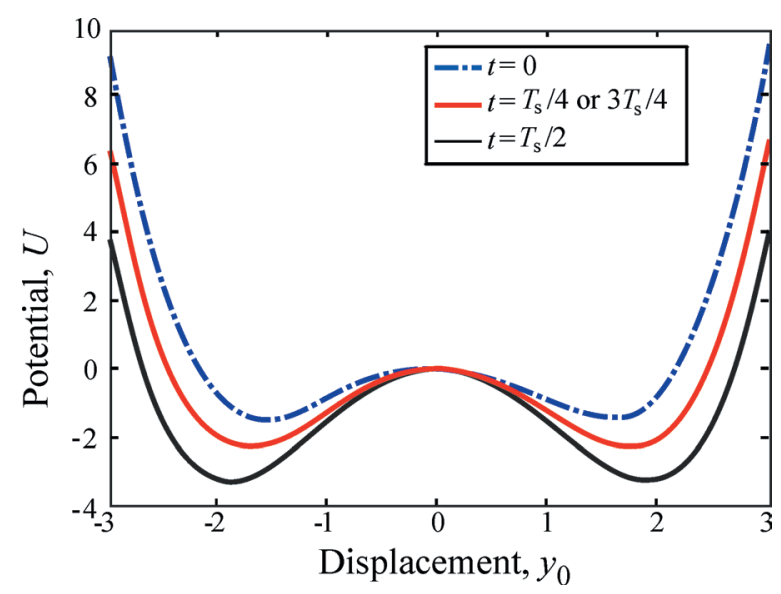

Fig. 2. The potential well when $\omega^{2}=3, \lambda=0.2$ and $b=1$

A statistical synchronization between the noiseinduced hopping and periodic excitation will take place, when the average waiting time $T_{\mathrm{w}}$ between two 
noise-induced inter-well transitions is comparable with half the period of the periodic excitation $T_{\mathrm{s}}$. The probability of a transition between the two potential wells is determined by the Kramers rate $r_{\mathrm{K}}$, and it will be small if the potential barrier between the two wells is very large. Therefore the waiting time $T_{\mathrm{w}}$ can be defined by the Kramers rate $r_{\mathrm{K}}$ as $T_{\mathrm{w}}=1 / r_{\mathrm{K}}$, and $r_{\mathrm{K}}$ for the model in this paper can be expressed by:

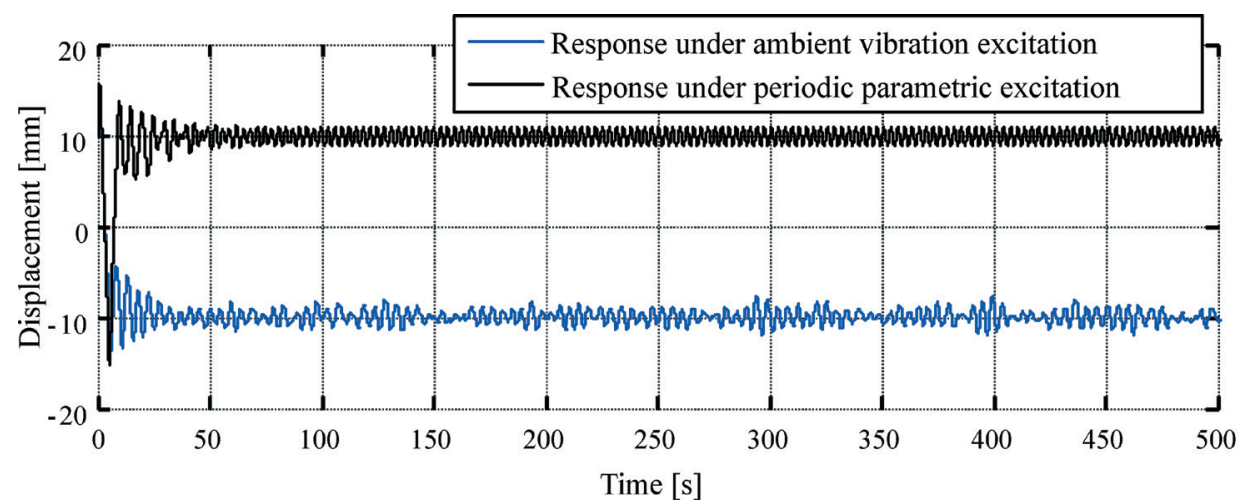

Fig. 3. Response under ambient vibration excitation, or periodic parametric excitation: the blue line fluctuating at -10 mm and the black line fluctuating at $10 \mathrm{~mm}$ represent the responses under the ambient vibration excitation and the periodic parametric excitation, respectively

a)

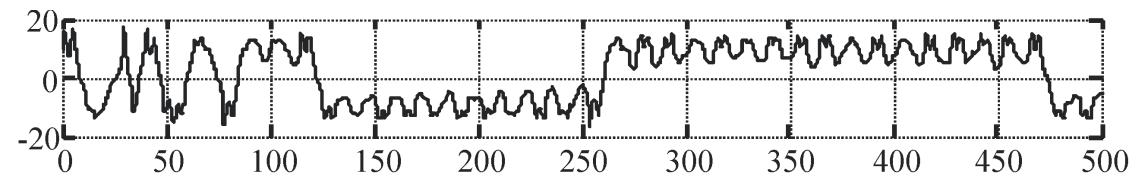

b)

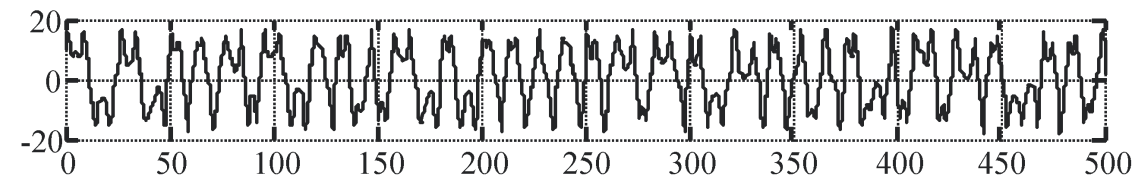

c)

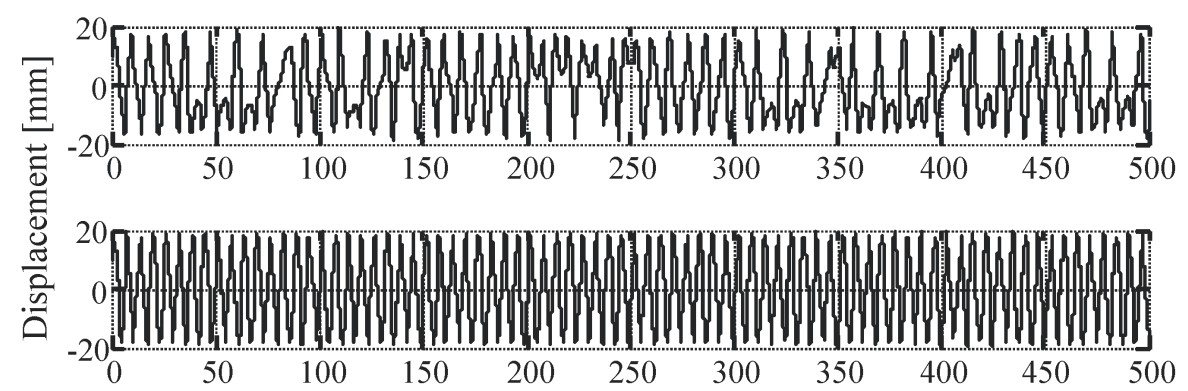

e)

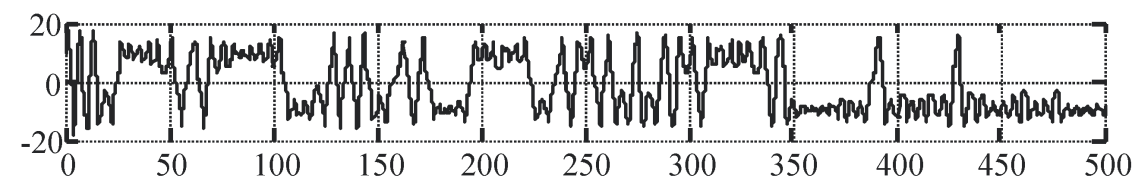

f)

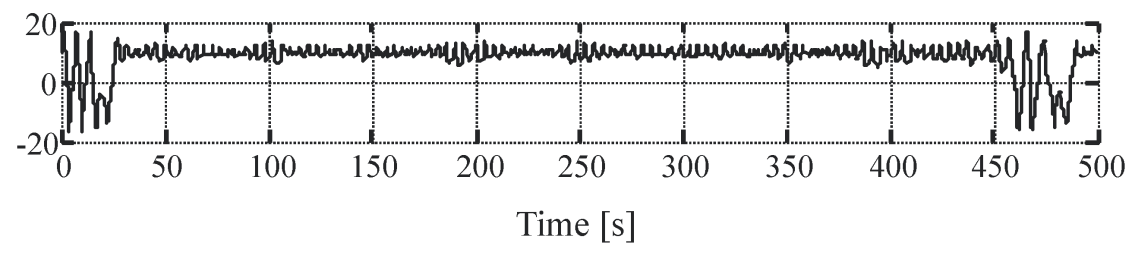

Fig. 4. Responses under ambient vibration excitation and parametric excitations at different frequencies for $\Omega$; a) 0.5, b) 1.0, c) 1.5 , d) 2.0 , e) 2.5 , and f) $3.0 \mathrm{rad} / \mathrm{s}$ 


$$
r_{K}=\frac{\omega^{2}}{\sqrt{2} \pi} \exp \left(-\frac{\omega^{4}}{D}\right),
$$

where $D$ is the intensity of the ambient vibration. For example when the ambient vibration is white noise this can be defined as $N(t)=\sqrt{2 D} g(t)$, where $g(t)$ is the Gauss function. Therefore the conditions for stochastic resonance defined by the frequency $f$ of the periodic excitation in $\mathrm{Hz}$ and noise strength can be transformed to the following,

$$
f=\frac{1}{2} r_{K}
$$

Contrary to this, when the excitation frequency in $\mathrm{Hz} f$ exceeds that value then stochastic resonance cannot occur.

According to Eq. (16), when $\omega^{2}=3$, the range of the excitation frequency $\Omega$ for stochastic resonance where $\Omega=2 \pi f$, is 1.98 to $2.12 \mathrm{rad} / \mathrm{s}$. In order to confirm that stochastic resonance can definitely occur under this condition, the numerical simulations have been undertaken. White noise acts on the system as the ambient vibration, simultaneously the excitation with different values of $\Omega$ is also input to the system. It should be noted that it is necessary to distinguish between the effects of stochastic resonance and normal resonance.

The blue line in Fig. 3 shows the displacement response of the end mass just under the ambient vibration excitation and it can be seen that the beam is unable to jump between the two potential wells. Conversely, the black line presents the displacement response for the parametric excitation on its own, operating at $1.7 \mathrm{~Hz}$. This excitation alone is unable to excite the beam into bi-stable vibration. In Fig. 4 , it can be seen that when the parametric periodic excitation and ambient vibration are input together, the system response increases. However, for different values of frequency $\Omega$, the response is seen to change. When $\Omega=0.5 \mathrm{rad} / \mathrm{s}$, the system behaves as a single stable state for most of the simulation time. When $\Omega$ increases to 1.0 and $1.5 \mathrm{rad} / \mathrm{s}$, the system behaves as the bistable state in a certain period. When the excitation frequency is $2.0 \mathrm{rad} / \mathrm{s}$ as shown in Fig. 4d, which is close to the theoretically predicted parametric excitation frequency for stochastic resonance, the large vibration is observed. The response is the strongest in the six cases shown in Fig. 4 and the system seems to be in the bistable state all the time. The response becomes weaker when $\Omega$ exceeds $2.0 \mathrm{rad} / \mathrm{s}$. From these results it can be concluded that the vibration is enhanced by the stochastic resonance.

\section{ENERGY HARVESTING}

For the energy harvester shown in Fig. 1, the piezoelectric film will change the damping radio of the system. According to the general model of a vibration energy harvester, the damping coefficient $c$ is represented by $c_{e}+c_{m}$, where $c_{m}$ is the mechanical damping coefficient, and $c_{e}$ represents the electrically induced damping coefficient. The system can harvest from the energy absorbed by the electrically induced damping. The dynamics of the system can be described by rearranging Eq. (13) as follows:

$$
\begin{aligned}
& \ddot{y}_{\mathrm{M}} \dot{y}_{\mathrm{M}}+\left(c_{e}+c_{m}\right) \dot{y}_{\mathrm{M}}^{2}+b y_{\mathrm{M}}^{3} \dot{y}_{\mathrm{M}}- \\
& -\omega^{2}(1+\lambda \cos \Omega t) y_{\mathrm{M}} \dot{y}_{\mathrm{M}}=N(t) \dot{y}_{\mathrm{M}} .
\end{aligned}
$$

Eq. (17) can be transformed to:

$$
\begin{aligned}
& \frac{d}{d t}\left(\frac{1}{2} \dot{y}_{\mathrm{M}}^{2}+\frac{\omega^{2}}{2} y_{\mathrm{M}}^{2}+\frac{b}{4} y_{\mathrm{M}}^{4}\right)+\left(c_{e}+c_{m}\right) \dot{y}_{\mathrm{M}}^{2}= \\
& =\dot{y}_{\mathrm{M}} N(t)+\lambda \omega^{2} \cos (\Omega t) y_{\mathrm{M}} \dot{y}_{\mathrm{M}} .
\end{aligned}
$$

Eq. (18) presents the instantaneous power input into the system, which is equal to the sum of the instantaneous power dissipated by damping and time derivative of the sum of the kinetic and potential energies. In the left hand side, $1 / 2 \dot{y}_{\mathrm{M}}^{2}$ is corresponding to the kinetic energy of the system, and $\omega^{2} / 2 y_{\mathrm{M}}^{2}+b / 4 y_{\mathrm{M}}^{4}$ presents the potential energy. And $\left(c_{e}+c_{m}\right) \dot{y}_{\mathrm{M}}^{2}$ is the instantaneous power absorbed by the damping, in which $c_{e} \dot{y}_{\mathrm{M}}^{2}$ is considered to be the energy that can be harvested. In the right hand side, $\dot{y}_{\mathrm{M}} N(t)$ and $\lambda \omega^{2} \cos (\Omega t) y_{\mathrm{M}} \dot{y}$ are corresponding to the energy supplied to the system by the ambient vibration and the periodic excitation, respectively. As the periodic excitation is the force need be supplied to produce the stochastic resonance, the energy $\lambda \omega^{2} \cos (\Omega t) y_{\mathrm{M}} \dot{y}_{\mathrm{M}}$ is considered to be the power consumed for the harvesting, while the energy $\dot{y}_{\mathrm{M}} N(t)$ is pure energy supplied from the ambient to the harvester.

When evaluating the performance of the energy harvesting, the energy $\lambda \omega^{2} \cos (\Omega t) y_{\mathrm{M}} \dot{y}_{\mathrm{M}}$ is extracted from the energy absorbed by the electric damping $c_{e} \dot{y}_{\mathrm{M}}^{2}$. Therefore the net instantaneous power which can be harvested can be given by:

$$
P=c_{e} \dot{y}_{0}^{2}-\left|\lambda \omega^{2} \cos (\Omega t) y_{0} \dot{y}_{0}\right|
$$

In order to investigate the enhancement of the energy harvesting by the stochastic resonance, a theoretical analysis with numerical simulation for the 

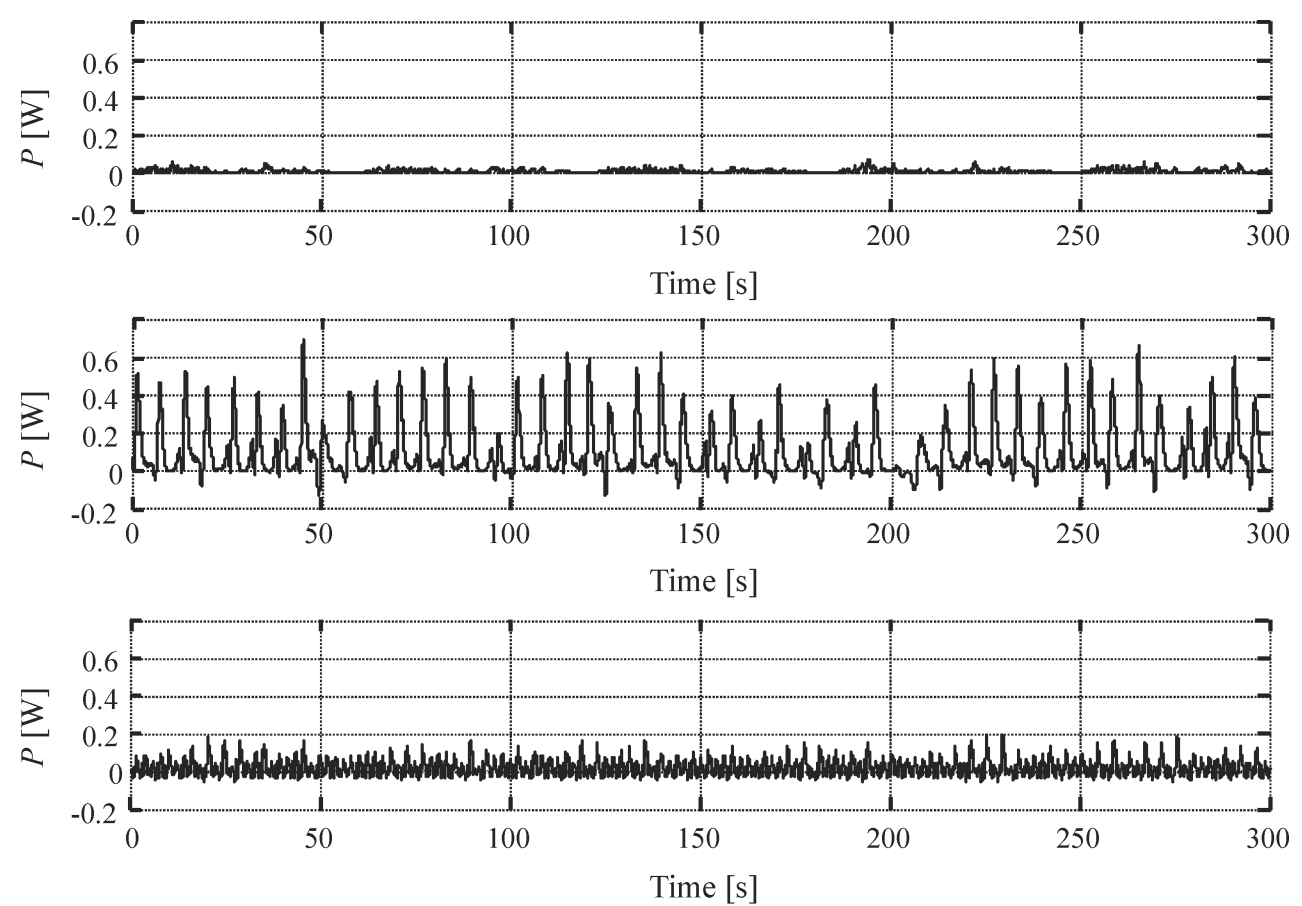

Fig. 5. Net available power under different conditions: a) without periodic excitation,b) stochastic resonance state, and c) natural resonance state

available power has been carried out. Fig. 5 represents the results of numerical simulations under the condition $c=0.5 \mathrm{Ns} / \mathrm{m}, c_{e}=0.3 \mathrm{Ns} / \mathrm{m}$, and $\omega^{2}=3 \mathrm{~s}^{-2}$. Fig. 5 shows the net powers generated when the periodic force is not supplied, when the periodic force whose angular frequency is $2.0 \mathrm{rad} / \mathrm{s}$, which is near the Kramers's rate, is supplied, and when the angular frequency is $1.7 \mathrm{~Hz}$, which is close to the natural frequency of the system, respectively. It is clear the system harvests the largest energy in the case of Fig. $5 \mathrm{~b}$. This indicates the stochastic resonance is the most effective way to generate energy from the ambient vibration. Furthermore, as we can adjust the frequency of the periodic excitation easily using the real time control unit, the stochastic resonance harvester can be operated in the best condition even if the characteristics of the ambient vibration changes. This is another advantage to the harvester using a typical resonance of the linear system.

\section{CONCLUSION}

A nonlinear mechanical vibration harvester that fulfills the conditions for stochastic resonance has been designed. Theoretical analysis has been implemented to illustrate the possibility of stochastic resonance in a certain mechanical vibration system. Furthermore, the feasibility to apply the stochastic resonance to the energy harvester has been investigated through numerical simulations. The effectiveness of stochastic resonance to enhance the amount of the energy to be harvested was evaluated, by comparing the harvested power with the case without the stochastic resonance. Although the power consumed in providing a periodic force reduces the total harvested power, the available power generated in stochastic resonance is obviously higher than the power generated from only ambient vibration. It is seen that stochastic resonance can act as a highly effective mean for energy harvesting from the ambient vibration.

\section{REFERENCES}

[1] Saha, C.R., O’Donnell, T.N., McCloskey, W.P. (2008). Electromagnet generator for harvesting energy from human motion. Sensors and Actuators A: Physical, vol. 147, no. 1, p. 248-253, DOI:10.1016/j.sna.2008.03.008.

[2] Dai, X., Wen, Y., Li, P., Yang, J., Li, M. (2011). Energy harvesting from mechanical vibrations using multiple magnetostrictive/piezoelectric composite transducers. Sensors and Actuators A: Physical, vol. 166, no. 1, p.94-101, DOI:10.1016/j.sna.2010.12.025.

[3] Chandrakasan, A., Amirtharajah, R., Goodman, J., Rabiner, W. (1998). Trends in low power digital signal processing. Proceedings of the IEEE International 
Symposium on Circuits and Systems, vol. 4, p. 604-607, DOI:10.1109/ISCAS.1998.699014.

[4] Mitcheson, P.D., Miao, P., Stark, B.H., Yeatman, E.M., Holmes, A.S., Green, T.C. (2004). MEMS electrostatic micro power generator for low frequency operation. Sensors and Actuators A: Physical, vol. 115, no. 2-3, p. 523-529, DOI:10.1016/j.sna.2004.04.026.

[5] Yoon, S., Lee, Y., Lee, S., Lee, C. (2008). Energyharvesting Characteristics of PZT-5A under gunfire shock. Materials Letters, vol. 62, no. 1-2, p. 36323635, DOI:10.1016/j.matlet.2008.04.042.

[6] Ferrari, M., Ferrari, V., Guizzetti, M., Andò, B., Baglio, S., Trigona, C. (2009). Improved energy harvesting from wideband vibrations by nonlinear piezoelectric converters. Procedia Chemistry, Proceedings of the Eurosensors XXIII conference, vol. 1, no. 1, p. 12031206, DOI:10.1016/j.proche.2009.07.300.

[7] Sari, I., Balkan, T., Kulah, H. (2008). An electromagnet micro power generator for wideband environmental vibrations. Sensors and Actuators A: Physical, vol. 145-146, p. 405-413, DOI:10.1016/j.sna.2007.11.021.

[8] Liu, J., Fang, H., Xu, Z., Mao, X., Shen, X., Chen, D., Liao, H., Cai, B. (2008). A MEMS-based piezoelectric power generator array for vibration energy harvesting. Microelectronics Journal, vol. 39, no. 5, p. 802-806, DOI:10.1016/j.mejo.2007.12.017.

[9] Mansour, M.O., Arafa, M.H., Megahed, S.M. (2010). Resonator with magnetically adjustable nature frequency for vibration energy harvesting. Sensors and Actuators A: Physical, vol. 163, no. 1, p. 297-303, DOI:10.1016/j.sna.2010.07.001.

[10] Zhu, D., Roberts, S., Tudor, M.J., Beeby, S.P. (2010). Design and experimental characterization of a tunable vibration-based electromagnet micro-generator. Sensors and Actuators A: Physical, vol. 158, no. 2, p. 284-293, DOI:10.1016/j.sna.2010.01.002.

[11] Ferrari, M., Ferrari, V., Guizzetti, M., Marioli, D. (2010). A single-magnet nonlinear piezoelectric converter for enhanced energy harvesting from random vibrations. Procedia Engineering, vol. 5, p. 1156-1159, DOI;10.1016/j.proeng.2010.09.316.
[12] Ferrari, M., Ferrari, V., Guizzetti, M., Andò, B., Baglio, S., Trigona, C. (2010). Improved energy harvesting from wideband vibrations by nonlinear piezoelectric converters. Sensors and Actuators A: Physical, vol. 162, no. 2, p. 425-431, DOI:10.1016/j.sna.2010.05.022.

[13] Gammaitoni, L., Hanggi, P., Jung, P., Marchesoni, F. (1998). Stochastic resonance. Reviews of Modern Physics, vol. 70, no. 1, p. 223-287, DOI:10.1103/ RevModPhys.70.223.

[14] Benzi, R., Parisi, G., Sutera, A., Vulpiani, A. (1982). Stochastic resonance in climatic change. Tellus, vol. 34, no. 1, p. 10-16, DOI:10.3402/tellusa.v34i1.10782.

[15] Klamecki, B.E. (2005). Use of stochastic resonance for enhancement of low-level vibration signal components, Mechanical Systems and Signal Processing, vol. 19, no. 2, p. 223-237, DOI:10.1016/j.ymssp.2004.03.006.

[16] Harmer, G.P., Davis, B.R., Abbott, D. (2002). A review of stochastic resonance: circuits and measurement. IEEE Transactions on Instrumentation and Measurement, vol. 51, no. 2, p. 299-309, DOI:10.1109/19.997828.

[17] Rallabandi, V.P., Roy, P.K. (2010). Magnetic resonance image enhancement using stochastic resonance in Fourier domain. Magnetic Resonance Imaging, vol. 28, no. 9, p. 1361-1373, DOI:10.1016/j.mri.2010.06.014.

[18] McInnes, C.R., Gorman, D.G., Cartmell, M.P. (2008). Enhanced vibrational energy harvesting using nonlinear stochastic resonance. Journal of Sound and Vibration, vol. 318, no. 4-5, p. 655-662, DOI:10.1016/j. jsv.2008.07.017.

[19] Hu, H., Nakano, K., Cartmell, M.P., Zheng, R., Ohori, M. (2012). An experimental study of stochastic resonance in a bistable mechanical system. Journal of Physics: Conference Series, vol. 382, DOI:10.1088/1742-6596/382/1/012024.

[20] Zheng, R., Nakano, K., Hu, H., Su, D., Cartmell, M.P. (2014). An application of stochastic resonance for energy harvesting in a bistable vibrating system. Journal of Sound and Vibration, vol. 333, no. 12, p. 2568-2587, DOI:10.1016/j.jsv.2014.01.020. 